\title{
NAIL DEFORMITIES DETECTION AND CLASSIFICATION USING IMAGE PROCESSING TECHNIQUE
}

\author{
KAMBAR PRIYANKA ${ }^{1} \&$ DR. PREETHI N PATIL ${ }^{2}$ \\ ${ }^{l} P G$ Student, Department of MCA, RV College of Engineering, Bangalore, India \\ ${ }^{2}$ Assistant Professor, Department of MCA, RV College of Engineering, Bangalore, India
}

\begin{abstract}
The different parts of the human body, are scanned in the deformities of the different effects. The analysis of human nails is a way to detect certain diseases in the human body. The nails are part of the body in which we can make a diagnosis, which is necessary for the use of your photos, nail diseases, diagnosis, and other tests and a scan. The final twist is one of the most natural tools for research, as it commonly used in Ayurveda medicine, in which the nails can be a strong indicator of perceived grievances that occur in the human body. Human nails convey useful information, complaint, or any nutritional imbalances, depending on the kind, texture, and color. A person, who has many systemic and dermatological diseases, is very easy to diagnose, the nails on both of the arms and legs of thorough research. Many nail diseases have the first appearance of many of the underlying systemic diseases. Changes in the color and/or shape of the nails and make the sign of many diseases, primarily affecting the nails and if we can make use of digital image processing techniques to determine the way a person moves to a point, and then we can get more accurate results, and is easy to predict with many diseases.

KEYWORDS: Images Nail Treatment, Early Detection, CNN (Convolutional Neural Network).
\end{abstract}

Received: May 24, 2021; Accepted: Jun 16, 2021; Published: Jun 05, 2021; Paper Id: IJCSEITRDEC20214

\section{INTRODUCTION}

Keratin is the building block of nails, which strengthens the tissues of nails and fingers. By looking at the nails, we can detect patients' health conditions. Some variations in nail colors, malignity, nail appearances and the presence of some particular problems tell us about diseases like diabetes, fungal infections, liver diseases, melanoma, and malnutrition, Anybody can possess these nail disorders. The latest research shows that around $25 \%$ of people possess leukonychia (a rare disorder), which happens because of vitamin deficiency. This is detected by observing the common changes in nails such as changing the color of nails to white, Thickening of nails, Changes in nail shape, swelling or redness around nails, Image comparing methodology, automatic melanoma detection, classification of dermatological images, and skin disease prediction are the latest trends. Deep Learning algorithms provided results asset side by side to the traditional methods like

Logistic Regression (LR), Support Vector Machine (SVM), Random Forest (RF), K-Nearest Neighbour $(\mathrm{KNN})$, Artificial Neural Network (ANN), Naive Bayes (NB), the problems detected by our algorithm is the scarcity of high-resolution images set for this process. We required the exact and standard set of images for computing the result. Regarding the liabilities, we have combated modern machine learning techniques and uses deep Convolutional Neural Networks Area under Curve (AUC) that calculated together with Receiver Operating Characteristic (ROC) curves. 


\section{PARTS OF A NAIL}

The keratogenic membrane matrix, nail matrix, and nail tissue lie in the part of the nail plate that runs under the root of the nail, and where the nerves, lymph, and blood vessels are located. The matrix is responsible for the production of cells that the lövhəli nail receives. The width and thickness of the nail plate were determined by the size of the length and thickness of the matrix, while the shape at the tip of the finger determines whether the nail plate straight, curved, and hooked. The array will suffer harm; it feeds and remains in a healthy state. As the new cells of the nail plate incubate, the pop-up matrix at the round table and the white color will push the old cells of the nail plate forward; and thus the old cells. The visible part of the matrix in a white crescent shaped pattern based on the visible nails. It is in the form of the area of the largest, thumb, and on the little finger, it is often omitted. The nail bed is the skin under the nails. Like the whole skin of these, two types of tissue: the deeper dermis, living tissue, bone, skin and blood vessels, and cancer, and the surface of the epidermis of the skin, a two-layer just under the nail plate movement. The epidermis is the angle to the dermis. The nail plate or body nail is the actual nail and so on for hair and skin, consisting of a translucent keratin protein that is made up of chains of amino acids. By bending the nails, it produces a powerful, flexible material consisting of several layers of dead flattened sign cells, visible as, pink and beyond lying it under kapilyarların function. The free edge or distal edge is an advanced nail plate corresponding to the abrasive or leading edge of the nail. Hyponychia is an epithelium located under the nail lövhəli at the junction between the free edge and the skin at the tip of the finger. It is safe to nail in bed. The onychodermal band is the seal between the lövhəli nail and the hyponychium. It is just under the free edge in the part of the nail where the nail ends in the bed and maybe taken in its glass, gray, color. It can be seen in some people, but others are very conspicuous. The last sin is a deep fat that will stick the root of the nail. The root of the nail is the part of the nail that stops, and the sinus functions, i.e. the main nail is connected to the lower part of the skin. This is an increase in the tissue below the matrix.

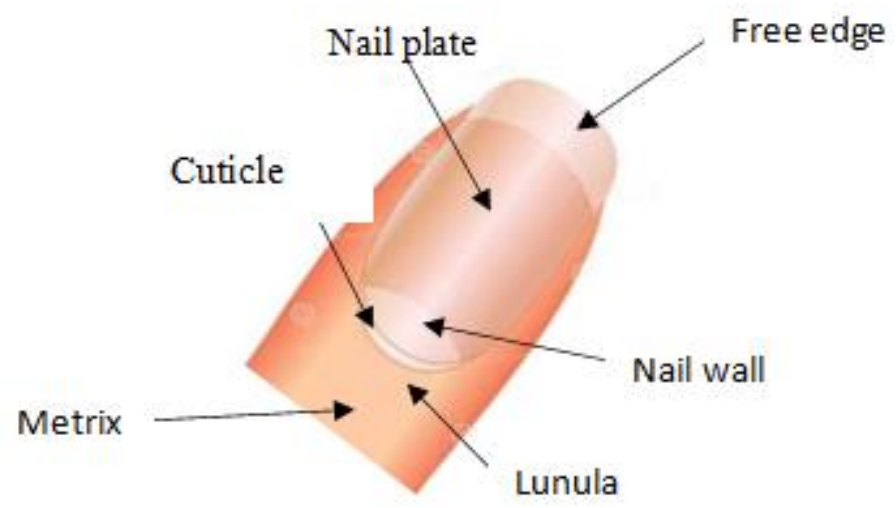

Figure 1: Parts of Nail.

Eponychia is a small band-a section of the epithelium of the back wall of the nail to the base of the nail. Often incorrectly referred to as a "proximal fold" or "cuticle", an eponychium is the end of a proximal fold that involves pouring, excess sebum over the newly formed nail plate's skin layer. These bearings are dry; you can say that in the invisible part of the skin and hair, which "leads" the upper part of the nail plate. Together, the eponychium and cuticle form a secure panel. The cuticle is a nail plate that is representing in non-viable cells and is eliminated during manicure, but the eponychium is a living cell that should not be touched. The perionyx is the protruding edge of the eponychium, which covers the proximal band of the shaped area. A nail wall is the tuk side of the skin and the proximal end of the nail. The side borders are located 
under the nail wall in the sides of the nails, and the nail groove or tuk is the skin of the gaps that, the side edges have themselves enough.

\section{HUMAN HEALTH AND FINGER NAIL}

Each finger represent group of organs, which summarize below

Table 1: Finger Relation with Body Parts.

\begin{tabular}{|l|l|}
\hline \multicolumn{1}{|c|}{ Finger name } & \multicolumn{1}{c|}{ Organ } \\
\hline Index finger & Gall bladder or Nervous system, Liver \\
\hline Middle finger & Heart and Circulatory system \\
\hline Ring finger & Reproductive and the Hormonal system \\
\hline Little finger & Digestive system \\
\hline The Thumb & Reproductive system and Excretory system, Brain \\
\hline
\end{tabular}

Generally, smooth, pink color and shining nails indicate a healthy human. The analysis of nails and their related disease symptoms are

Table 2: Nail Disorders and Related Disease

\begin{tabular}{|l|l|}
\hline \multicolumn{1}{|c|}{ Category } & \multicolumn{1}{c|}{ Nail Disease } \\
\hline Dematosis & Onycholysis, Splinter Haemorrhage, Darier's disease, Alopecia \\
\hline Change in shape & Clubbing, Koilonychia \\
\hline Change in surface & Bau's lines, Meruhrcke's line, Leuonychia \\
\hline Change in color & $\begin{array}{l}\text { Terry's nails, linday's } \\
\text { Nails, Red lunula, Splinter Haemorrhage, Yellow nail syndrome. }\end{array}$ \\
\hline
\end{tabular}

\section{LITERATURE SURVEY}

This study uses the Convolutional Neural Network (CNN) method. The CNN method, which is used to provide good performance under many image classification factors [4]. A CNN method that is designed to identify two-dimensional shapes, providing a high level of inversion for broadcasting, scaling, and other violations. Currently, many scientists are working in disease identification, Like the Extraction of features and Allocation to create exact results on nail images to identify the disease; Many Researchers have pretended to apply both deep learning and machine learning algorithms recently [1]. To create an exact arrangement of Leukonychia disease from an image dataset, a lot of researchers have pretended to apply both deep learning and machine learning algorithms [2]. This technique involves the induction of (ANN, CNN, RF, SVM, NB, LR, CN2, and the AdaBoost rules [3]. This section describes the process of performing a variety of Simulations, for the purpose of classification of leukonychia certain amount of information, photos, [5] AlexNet structure, that is, to learn how to classify the various nail diseases. Where, exactly, RELU uses a sigmoid function, to obtain a good degree of accuracy. Pandit et al. [6] investigated the characteristics, photos, and prognosis of the disease with the help of the data from the medical palmistry. Therefore, by our analysis, it can be a skeleton of CUPS, created for the primary leukonychia in an age in which so happens to be the exact 93.8\%. [7]. In this section, he will give us insights on the variety of diseases affecting nails based on previous work in digital image processing for this disease, on the prognosis, on various features isolated from nails, and on the classification method [8]. You can predict a variety of diseases during each of them by studying human nails. The offer of crosshair Hair and an image processing system using KNN (NIP-N), allows us to create models that you can analyze, person, nail, and thus help us predict various diseases. At the entrance to 
the system manually, enter a Photo in yourself. [9] The method continues with the introduction of the photo segmentation method and then performs analysis for the region nailed to it. This is the only process like BMP, GIF, JPEG, PNG, TIFF image file formats. It can detect two or three diseases.

\section{METHODOLOGY}

In this study, this method is used in a convolutional neural network (CNN). This method is designed to recognize twodimensional forms of software, with high-level inversion for translation, scaling, and other violations. CNN includes a kind of deep neural network based on a high-level in-depth network very much applied to image data [6]. In general, a convolutional neural network is divided into two main parts: a feature removal layer and a fully connected layer. Apple Features-itself consists of a convolutional layer that combines inventory and classification layers. But there have been many changes in various CNN architectures, because VGGNet, GoogLeNet, ReLU, BN-Inception, Inception-v3, post [10] explains the impact on existing architectures. After that, experiments in this study, we will analyze CNN to find nail anomalies. In the next proposed system, we used the CNN Discrete function equations it has many layers and these layers convolution operations. are carried out between the input image and a kernel at all points and its max and min pooling results using two matrices, and it converts the input image into a smaller size to reduce the number of parameters with a sampling operator[11], and figer3 tells about the hidden layers and main output/ results. In addition, we in our proposed method simplified usage and end result free of many complications by adding all those data, which be available to the analytical board which thru mat-lab, the image processing brings $99.9 \%$ results.

\section{PROPOSED APPROACH}

CNNs are deep learning techniques often applied to a two-dimensional image. This approach has two main parts, which are the convolution Layer, a combination of coat and classification society, which gives the layer a convolution layer base layer, which is based on the network, where these components of the convolution operation should take the picture, and all the main elements. This process can use discrete function equations $\mathbf{f}(\mathbf{n}, \mathbf{m})$ and $\mathbf{h}(\mathbf{n}, \mathbf{m})$ which is defined by

$$
\begin{array}{cc}
\text { Tn } & \text { Tm } \\
y(x 1, x 2)= & \sum \sum f(x 1+k 2, m) h(n, m) \\
n-1 & m-1
\end{array}
$$

The illustration of the equation above can see in the following picture:

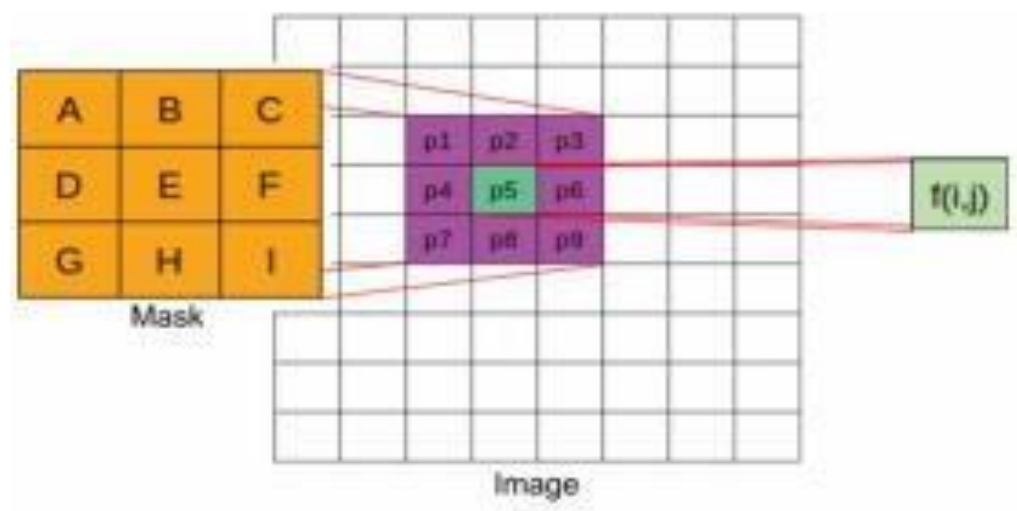

Figure 1: Convolution Illustration 
This is followed by the merge process, which is the process used to transform a small input image. It is designed to reduce the input space (by reducing the number of parameters), operation through the lower selection. There are two concatenation bearings used in the architectural Inception-v3, i.e. Maximum Pool and Average Pool. Maximum community is a mathematical operation, i.e. the job is to get the maximum value of a part of an image of a certain size, at the same time, i.e. the average value of a combination is a mathematical operation, i.e. the job is to get the average value of a part of an image of A certain size.
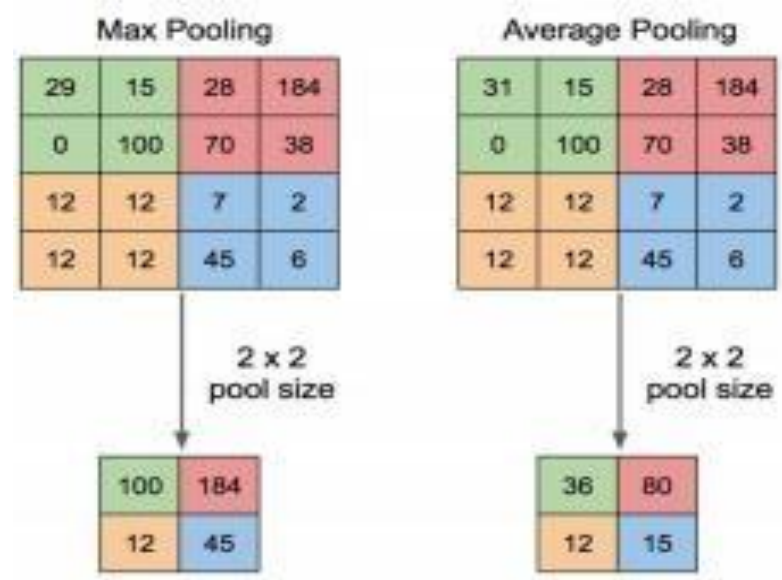

Figure 2: Illustration of Max Pooling and Average Pooling

Shown in the photo above is an example of the maximum reconciliation of operations and the average value, the filter size is $2 \times 2$ pixel $4 \times 4$ pixels of the input signal. The maximum merge, each filter, taking the maximum value, and then rebuilt, a new generation, with a size of $2 \times 2$ pixels. And this is the average cost of merges, the cost of which is on average worth the filter size. Competition classification layer is a layer consisting of flattened, hidden layers, and activate features. Hidden layers of artificial neural networks are the layer between the input layer and the rest layer of artificial neurons that have a set of input weights and produce output through activation of features.

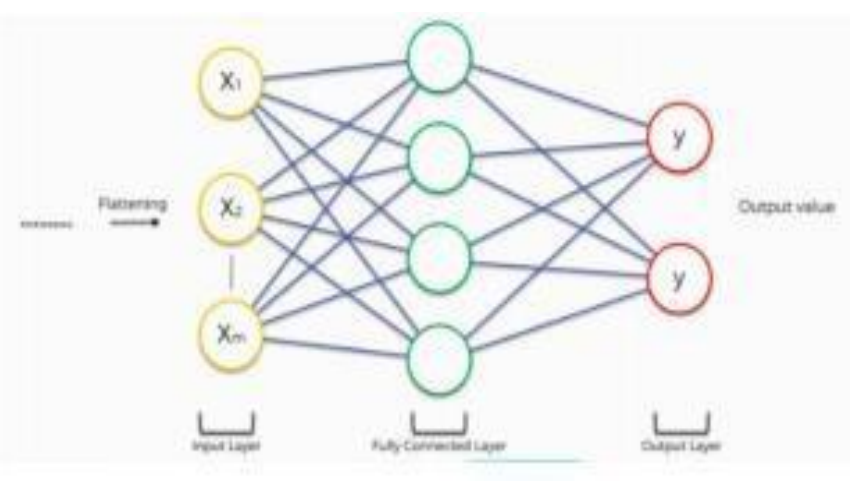

Figure 3: Hidden Layer

\section{EXPERIMENT}

Before classification, Simple image processing techniques automatically re-processes all images in the dataset. For each class image, the digits 0 and 1 are encoded, where 0 represents fresh and healthy nails, and 1 represents Terry's nails (aloperia areata) disease. Next, all nail images are resized in $224 \times 224$ pixels. Then the image that is divide into training data, testing data and data validation. In addition, the data set we taken in to two types 


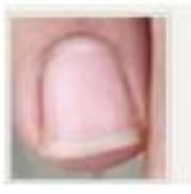

Healthy nails

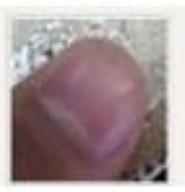

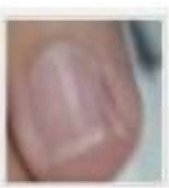

N.

Figure 4: The Nai terry's nails
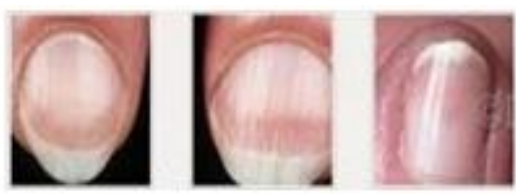

Feature is Shown

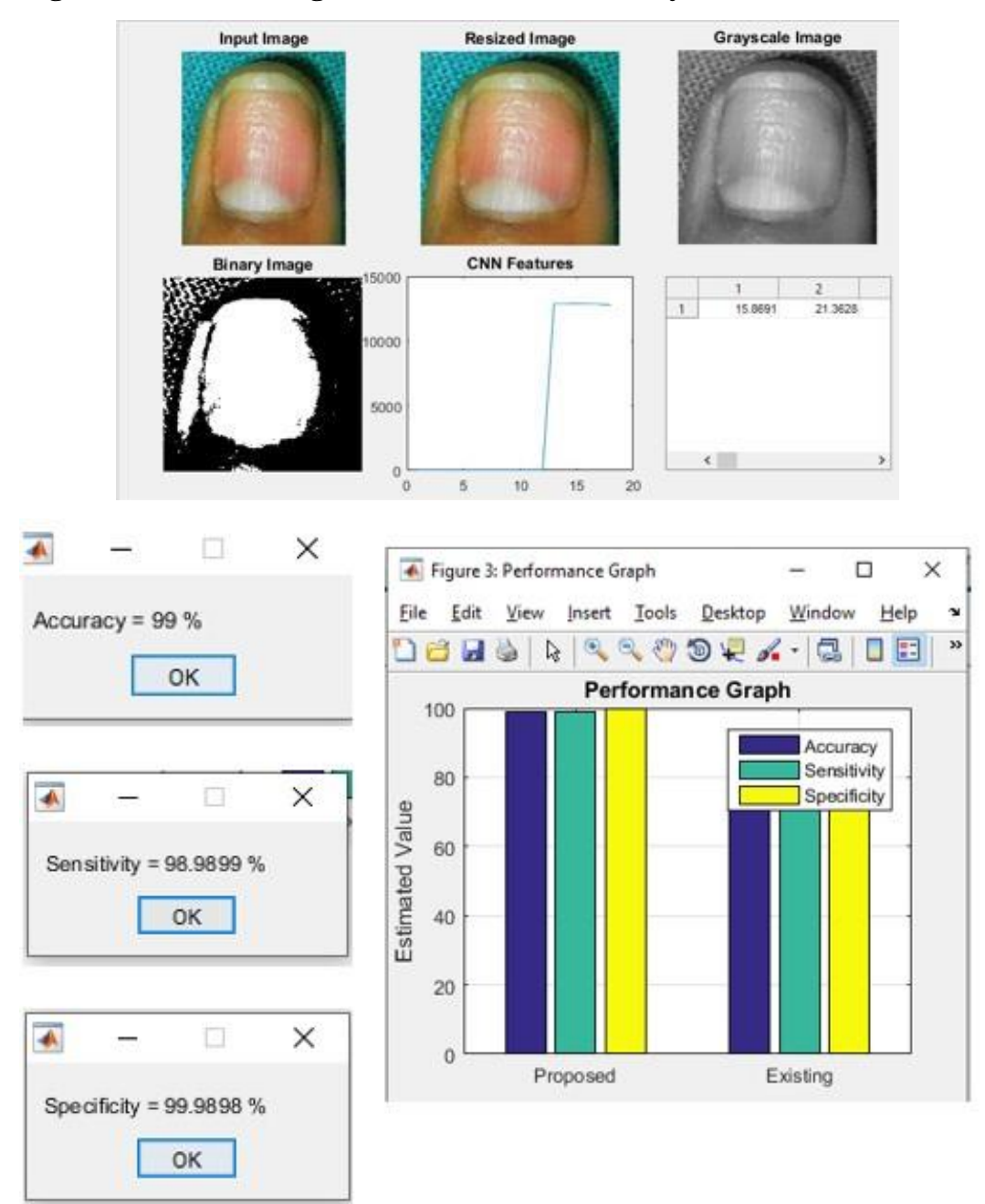

Figure 5: Performance Graph and Some Results

\section{CONCLUSIONS}

Currently, a non-invasive procedure for diagnosing the disease is an important part of the healthcare sector. Various functions in the human body, and a mechanism that can be useful for non-invasive treatment, diagnosis of diseases like a nail, picture, eye, respiratory, etc. human spike image analysis, non-invasive procedures where various image processing methods such as photography, primary processing, images, image segmentation extract features used analysis for hair. Various methods, classification as SVM group, KNN group, ANN classification. CNN various diseases that are diagnosed, spike analysis. By the different hair, and features like nail polish, nail shape, In our methodology, we used Convolutional Neural Network to predict affected nails that are predicted from data images obtained result of $93.8 \%$ correctness. Because of the scarcity of an already present standard set of data, having a large quantity of image dataset of nail images, as we 
know there is still some opportunity for continuation in the nearest future. We are looking to develop better accurate and specific methods used in the computation of probability.

\section{REFERENCES}

1. Aishwarya, \& Goel, Akansha \& Nijhawan, Rahul. (2019). A Deep Learning Approach for Classification of Onychomycosis Nail Disease. 10.1007/978-3-030-30577-2_98.

2. Basavaraj Hiremath \& SC Prasannakumar, "Automated Evaluation of Breast Cancer Detection Using SVM Classifier", International Journal of Computer Science Engineering and Information Technology Research (IJCSEITR) ,Vol. 5, Issue 1,pp, $11-20$

3. S. Gandhat, S. Avhad, R. Alawadh, and N. Bajpai, "Study and Analysis of Nail Images of Patients," Int. J. Comput. Appl., vol. 143, no. 13, pp. 38-41, 2016.

4. Mohammad Omid Khairandish, Ruchika Gupta \& Meenakshi Sharma, "A Hybrid Model of Faster R-CNN and SVM for Tumor Detection and Classification of MRI Brain Images", International Journal of Mechanical and Production Engineering Research and Development (IJMPERD), Vol. 10, Issue 3,pp, 6863-6876

5. H. A. L. M. Usawi, “- Infectious Diseases,” vol. 16, no. 3, pp. 311-312, 2012.

6. Y. A. Gunge and T. S. Indi, "Early Stage Disease Diagnosis System Using Human Nail Image Processing," Int. J. Inf. Technol. Comput. Sci., vol. 8, no. 7, pp. 30-35, 2016.

7. Shaik. Bajid Vali, A. K. Sharma \& Syed Musthak Ahmed, "Significance of Images and Algorithms of Image Processing for Detection and Determination of Diabetes at Early Stage", International Journal of Electronics and Communication Engineering (IJECE) ,Vol. 6, Issue 5,pp; 17-28

8. Nijhawan, Rahul \& Joshi, Deepankar \& Narang, Naman \& Mittal, Aditya \& Mittal, Ankush. (2019). A Futuristic Deep Learning Framework Approach for Land Use-Land Cover Classification Using Remote Sensing Imagery. 10.1007/978-98113-0680-8_9.

9. R. Nijhawan, R. Verma, Ayushi, S. Bhushan, R. Dua and A. Mittal, "An Integrated Deep Learning Framework Approach for Nail Disease Identification," 2017 13th International Conference on Signal-Image Technology \& Internet-Based Systems (SITIS), Jaipur, 2017, pp. 197-202

10. M. Dhivya, S. Deepa, S. Anbumani \& R. Kavin, "Detection and Grading of Cancer Using Histopathological Image Analysis", International Journal of Electronics and Communication Engineering (IJECE) ,Vol. 3, Issue 2,pp, 59-68

11. Nijhawan, Rahul \& Jindal, Radhika \& Sharma, Himanshu \& Raman, Balasubramanian \& Das, Josodhir. (2019). A Deep Learning Framework Approach for Urban Area Classification Using Remote Sensing Data. 10.1007/978-981-32-9088-4_37

12. Suartika, R. Soelaiman and Y. Wijaya, Arya, "Klasifikasi Citra Menggunakan Convolutional Neural Network (Cnn) Pada Caltech 101", J. Tek. ITS, vol. 5, no. 1, pp. 65- 69, 2016.

13. C. Szegedy, V. Vanhoucke, J. Shlens, and Z. Wojna, "Rethinking the Inception Architecture for Computer Vision," 2014.

14. Fujishima. Fingernail Detection Method from Hand Images including Palm. MVA2013 IAPR International Conference on Machine Vision Applications, May 20-[11]2013, Kyoto, JAPAN.

15. Gandhat S., Thakare A.D., Avhad S., et al. Study and Analysis of Nail Images of Patients. International Journal of Computer Applications (0975-8887), 143(13), June 2016 
16. Sharma V., Shrivastava A. System for Disease detection by analyzing finger nails Color and Texture. International Journal of Advanced Engineering Research and Science (IJAERS), Oct 2015, 2(10), ISSN: 2349-6495.

17. Yoonsik Kim, Insung Hwang, Nam Ik Cho. "Convolutional Neural Networks and Training Strategies for Skin Detection," INMC, Dept. of Electrical and Computer Engineering Seoul National University, Seoul, Korea.

18. Kumuda S. An Image Pre-processing Method for Fingernail Segmentation. 2017 IEEE 2nd International Conference on Signal and Image Processing, 978-1-5386-0969-9/17/\$31.00 @2017 IEEE

19. Saranya V., Ranichitra A. Image segmentation techniques to detect nail abnormalities” International Journal of Computer Technology \& Applications, 8(4), 522-527p.

20. Pandit H., Shah D.M. The Model for Extracting a Portion of a Given Image Using Color Processing. International Journal of Engineering Research \& Technology (IJERT), December 2012, 1(10), ISSN: 2278-0181.

21. Ramya V., Lydia M.A. Leaf Disease Detection and Classification using Neural Networks. International Journal of Advanced Research in Computer and Communication Engineering, November 2016, ISO 3297:2007 Certified 